\title{
THE NETWORK PERFORMANCE OF MULTI-RATE FDD-MODE UMTS
}

\author{
J.S. Blogh, L. Hanzo \\ Dept. of Electronics and Computer Science, Univ. of Southampton, SO17 1BJ, UK. \\ Tel: +44-2380-593 125, Fax: +44-2380-593 045 \\ Email: lh@ecs.soton.ac.uk; http://www-mobile.ecs.soton.ac.uk
}

\begin{abstract}
The performance of a multi-rate mobile cellular network using the Frequency Division Duplex (FDD) mode of the Code Division Multiple Access (CDMA) based Universal Mobile Telecommunication System (UMTS) is investigated. The new call blocking and call dropping probabilities and the tolerable network load are studied in the context of a multi-rate FDD-mode UMTS network incorporating dynamic threshold assisted soft handovers and shadow fading.
\end{abstract}

\section{INTRODUCTION AND SOFT HANDOVERS}

The standardisation of the third-generation (3G) systems, such as UMTS, is reaching a state of maturity [1-5]. Hence it is beneficial to study the associated network performance, which is the objective of this contribution.

The process of soft handovers is based on a make-beforebreak approach, where a new communications link is established before the existing link is relinquished due to the associated link quality degradation. The mobile station (MS) continuously monitors the power level of the received PIlot CHannels (PICH) transmitted from the neighbouring basestations (BSs). The power levels of these basestations are compared against two thresholds, $T_{a c c}$ and $T_{d r o p}$. If the power level is above the basestation's acceptance threshold, $T_{a c c}$, then assuming the basestation is not already in the Active Basestation Set (ABS), it is added to the ABS. If, however, the PICH of a basestation in the ABS is found to be below the dropping threshold, $T_{d r o p}$, then the basestation is removed from the ABS. If the threshold $T_{a c c}$ is set to too low a value, then basestations are added unnecessarily to the ABS, which results in extraneous network resource utilisation. Conversely, if $T_{a c c}$ is excessively high, then it is possible that no basestations may exist within the ABS at the cell extremities. A mobile station is in simultaneous communication with two or more basestations during the soft handover, hence optimal combining of the downlink signals of several BSs is performed at the MS.

By contrast, the network invokes selective combining of the MSs' signals decoded at each basestation. Since a dropped call is less desirable from the user's point of view,

The financial support of the following organisations is gratefully acknowledged: Engineering and Physical Sciences Research Council, Swindon, UK. than a blocked call, two resource allocation queues were invoked, one for new calls and the other - higher priority - queue, for handovers. By forming a queue of the handover requests, which have a higher priority during contention for network resources than new calls, it is possible to reduce the number of dropped calls at the expense of an increased blocked call probability. A further advantage of the Handover Queueing System (HQS) is that during the time, while a handover is in the queue, previously allocated resources may become available, hence increasing the probability of a successful handover.

A disadvantage of using fixed handover thresholds is that in some locations all the pilot signals may be weak, whereas in other locations they may all be strong. Hence, dynamic thresholds are advantageous. An additional benefit of using dynamic thresholds is experienced in a fading environment, where the received pilot strength may drop momentarily below a fixed threshold and thus may cause an ABS removal and addition. However, this basestation may be the only basestation in the ABS, which would result in a dropped call. Using dynamic thresholds this scenario would not have occurred, since the pilot strength would not have dropped below that of any of the other pilot signals.

\section{POWER CONTROL}

Accurate power control is essential in CDMA in order to mitigate the near-far problem, which affects the network capacity and coverage. Closed-loop power control is employed on both the uplink and downlink. The mobiles and basestations estimate the Signal-to-Interference Ratio (SIR) every $0.667 \mathrm{~ms}$, or in each timeslot, and compare this estimated SIR to a target SIR. If the estimated SIR is higher than the target SIR, then the relevant transmitter is instructed to reduce its transmit power. Likewise, if the estimated SIR is lower than the target SIR, the associated transmitter is instructed to increase its transmit power. Transmitting at an unnecessarily high power increases the power consumption and degrades the other users' signal quality by inflicting excessive co-channel interference. Hence, the other users may request a power increase in an effort to maintain their target link quality, potentially leading to an unstable system.

If the mobile is in soft handover, and therefore basestationdiversity combining is performed, then the basestations' transmit powers are controlled independently. Hence, the mobile station may receive different power control commands from the BSs in its ABS. Thus, the mobile only 
increases its transmit power, if all of the BSs in the ABS instruct it to do so. However, if any one of the basestations in the ABS instructs the mobile to decrease its power, then the mobile will reduce its transmit power. This method ensures that the multi-user interference is kept to a minimum, since at least one basestation has a sufficiently high quality link.

\section{CODE Allocation}

Again, an overview of the UTRA standard can be found for example in $[1,2,4,10]$. The downlink is assumed to be synchronous under the control of a basestation, however, the basestations are asynchronous with respect to the other basestations. The UMTS channelization codes are known as Orthogonal Variable Spreading Factor (OVSF) codes, which provide total isolation between different users on the synchronous downlink under perfect channel conditions, thus perfectly eliminating intra-cell Multiple Access Interference (MAI). However, the OVSF codes exhibit poor asynchronous cross-correlation properties and hence the inter-cell MAI may be high, unless the same code is only allocated to BSs exhibiting a sufficiently high geographic separation. By contrast, other codes such as Gold codes, exhibit a low asynchronous cross-correlation. Therefore, cellspecific long codes are used for reducing the inter-cell interference on the downlink. These so-called scrambling codes are Gold codes of $2^{18}-1$ chip-duration and each user served by a given basestation has the same downlink scrambling code. There are a total of 512 scrambling codes, potentially allowing the system to assign a different cell-specific scambling code to 512 cell sites, which eases the task of code planning and allocation.

The downlink OVSF codes are allocated by the basestation, again, facilitating perfectly interference-free isolation between different users on the synchronous downlink, if the channel coditions are perfect. Thus each user supported by a given basestation has a different downlink OVSF code, while MSs served by a different basestation may be using the same OVSF code.

On the asynchronous uplink the MAI is reduced by assigning different scrambling codes to different users, emphasizing again that the employment of scrambling codes exhibiting low asynchronous cross-correlation is important. The primary scrambling code is constructed from the socalled extended VL-Kasami code set [2] of length 256, where the short length enables low complexity multi-user detection [6] to be implemented.

For single-user detector assisted basestations, a long secondary scrambling code is used $[1,2,4,10]$, which is a Goldcode having a length of $2^{41}-1$ chips. Since the uplink transmissions are asynchronous and hence each user has a unique Gold code exhibiting a high asynchronous cross-correlation, every user can employ the same set of channelization codes.

\section{SIMULATING AN INFINITE PLANE NETWORK}

A tessellating rhombic simulation area of 7 cells by 7 cells was used, as shown in Figure 1, thus allowing the simulation

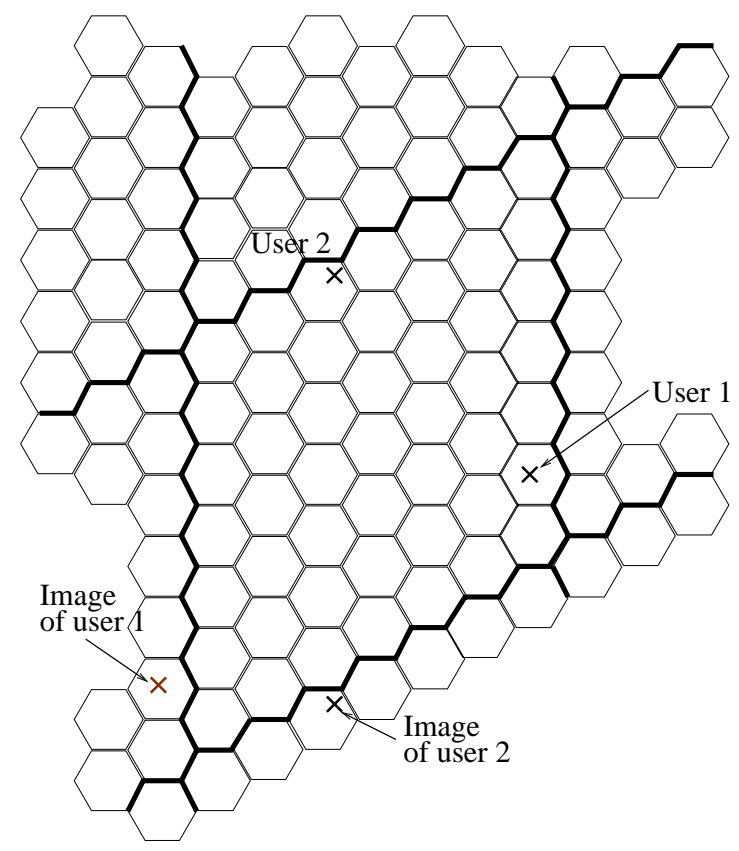

Figure 1: The $7 \times 7$ rhombic simulation area showing a user and its "wrapped" image.

area to be replicated around itself. This approach avoids the border or edge effects associated with simulating a "desertisland-like" cellular network, which results in the central cells experiencing significantly higher interference, than the edge cells. A further advantage is that all cells encounter a somewhat higher level of interference, than that experienced by an "island-type" system. Thus a higher call dropping rate occurs across the network, allowing statistically valid results to be obtained within a reduced-duration simulation time frame, hence expediting our simulations. The infinite plane network replicates itself, such that when a mobile leaves the central network, it enters an adjacent network which is mapped onto the central network. More explicitly, as a mobile leaves the network, it is "wrapped around" and enters the network at the opposite edge, whilst inflicting cochannel interference to all users of the network, who may be located at either edge of the network. In order to enable a call to be maintained under these conditions, both the signals and the interference are "wrapped around" the edges of the network.

\section{SYSTEM PARAMETERS}

New call channel allocation requests were placed in a resource allocation queue for up to $5 \mathrm{~s}$. If during this period a call was not not serviced, it was classed as blocked. The mobiles moved freely, in random directions, at a speed of $30 \mathrm{mph}$ within the simulation area, which consisted of 49 cells, as described in Section 4. The cell-radius was $218 \mathrm{~m}$. The call duration and inter-call periods were Poisson distributed with the mean values shown in Table 1. For our initial investigations we have assumed that the basestations and mobiles form a synchronous network, both in the up- 
and the down-link.

Furthermore, the basestations are assumed to be equipped with the Minimum Mean Squared Error Block Decision Feedback Equaliser (MMSE-BDFE) based Multi-User Detector (MUD) [6]. The post despreading SINRs required by this MUD for obtaining the target BERs were determined with the aid of physical-layer simulations using a 4-QAM modulation scheme, in conjunction with $1 / 2$ rate turbo coding and MUD over a COST 207 seven-path Bad Urban channel [8]. Using this turbo-coded MUD-assisted transceiver and a spreading factor of 16 , the post-de-spreading SINR required for maintaining the target BER of $1 \times 10^{-3}$ was $8.0 \mathrm{~dB}$. The BER corresponding to low-quality access was stipulated at $5 \times 10^{-3}$. This BER was exceeded for SINRs below 7.0dB. Furthermore, a low-quality outage was declared, when the BER of $1 \times 10^{-2}$ was exceeded, namely for SINRs below 6.6 dB. These values can be seen along with the other system parameters in Table 1.

\section{PERFORMANCE METRICS}

There are several performance metrics that can be used for quantifying the performance or quality of service provided by a mobile cellular network. The following performance metrics have been widely used in the literature and were also advocated by Chuang [7]:

- New call blocking probability, $P_{B}$.

- Call dropping or forced termination probability, $P_{F T}$. A call is dropped when the lower of the uplink and downlink SINRs dips consecutively below the outage SINR (5\% BER) a given number of times.

- Probability of a low quality access, $P_{l o w}$, quantifies the chances of either the uplink or downlink signal quality being sufficiently poor, resulting in a low quality access (1\% BER).

- Probability of outage, $P_{\text {out }}$, is defined as the probability that the SINR is below the value at which the call is deemed to be in outage.

- Grade-Of-Service (GOS) was defined by Cheng and Chuang [7] as :

$$
\begin{aligned}
G O S= & P\{\text { unsuccessful or low-quality call accesses }\} \\
= & P\{\text { call is blocked }\}+P\{\text { call is admitted }\} \times \\
& P\{\text { low signal quality and call is admitted }\} \\
= & P_{B}+\left(1-P_{B}\right) P_{\text {low }} .
\end{aligned}
$$

\section{SIMULATION RESULTS}

In order to determine the number of users that may be supported with adequate call quality by the network, we have defined a conservative and a lenient scenario which are formed from a combination of the performance metrics, as follows [9]:

- Conservative scenario : $P_{B} \leq 3 \%, P_{F T} \leq 1 \%, P_{l o w} \leq 1 \%$ and $G O S \leq 4 \%$.

- Lenient scenario : $P_{B} \leq 5 \%, P_{F T} \leq 1 \%, P_{l o w} \leq 2 \%$ and $G O S \leq 6 \%$.

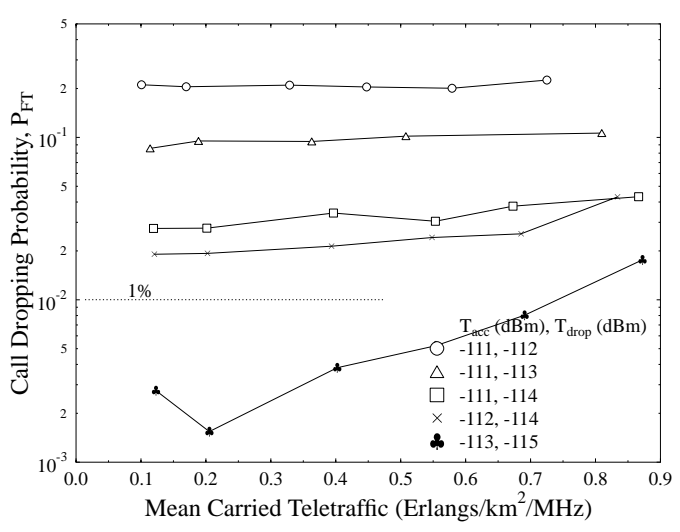

Figure 2: Call dropping probability versus mean carried traffic of a CDMA based cellular network using fixed received pilot power based soft handover thresholds in conjunction with $0.5 \mathrm{~Hz}$ shadowing having a standard deviation of $3 \mathrm{~dB}$ for $\mathrm{SF}=16$.

\subsection{Fixed Received Pilot Power Based Handover}

Our simplest handover algorithm studied in this contribution is based on the acceptance and dropping thresholds, $T_{a c c}$ and $T_{d r o p}$, respectively. In simple terms, a call is accepted and serviced, when the received pilot power is in excess of the threshold $T_{a c c}$ and dropped, when the corresponding pilot power falls below $T_{d r o p}$. In this section we examine the achievable performance, upon using fixed received pilot power based soft handover thresholds of $T_{a c c}$ and $T_{d r o p}$, when the MSs are subjected to log-normal shadow fading having a standard deviation of $3 \mathrm{~dB}$ and a maximum shadow-fading frequency of $0.5 \mathrm{~Hz}$.

The call dropping results of Figure 2 suggested that the network's performance was poor when using fixed received pilot power based soft handover thresholds in the above mentioned shadow fading environment. The root cause of the problem is that the fixed thresholds must be set such that the received pilot signals, even when subjected to shadow fading, are retained in the active set. Therefore, setting the thresholds too high results in the base stations being removed from the active set, thus leading to an excessive number of dropped calls. However, if the thresholds are set too low, in order to counteract this phenomenon, then the base stations can be in soft handover for too high a proportion of time, and thus an unacceptable level of low quality accesses is generated due to the additional co-channel interference inflicted by the high number of active base stations. Figure 2 shows that reducing the soft handover thresholds improved the network's call dropping probability, but Figure 3 illustrates that reducing the soft handover thresholds engendered an increase in the probability of a low quality access.

The network cannot satisfy the quality requirements of the conservative scenario, namely that of maintaining a call dropping probability of $1 \%$ combined with a maximum probability of low quality access below $1 \%$. However, the entire network supported 127 users, whilst meeting the le- 


\begin{tabular}{|l|r||l|r|}
\hline Parameter & Value & Parameter & Value \\
\hline \hline Noisefloor & $-100 \mathrm{dBm}$ & Pilot power & $1 \mathrm{dBm}$ \\
Frame length & $10 \mathrm{~ms}$ & Cell radius & $218 \mathrm{~m}$ \\
Multiple access & FDD/CDMA & Number of basestations & 49 \\
Modulation scheme & 4QAM/QPSK & Spreading factor & 16 \\
Minimum BS transmit power & $-44 \mathrm{dBm}$ & Minimum MS transmit power & $-44 \mathrm{dBm}$ \\
Maximum BS transmit power & $21 \mathrm{dBm}$ & Maximum MS transmit power & $21 \mathrm{dBm}$ \\
Power control stepsize & $1 \mathrm{~dB}$ & Power control hysteresis & $1 \mathrm{~dB}$ \\
Low quality access (0.5 \% BER) SINR & $7.0 \mathrm{~dB}$ & Outage (1\% BER) SINR & $6.6 \mathrm{~dB}$ \\
Pathloss exponent & -3.5 & Size of Active Basestation Set (ABS) & 2 \\
Average inter-call-time & $300 \mathrm{~s}$ & Max. new-call queue-time & $5 \mathrm{~s}$ \\
Average call length & $60 \mathrm{~s}$ & MS speed & $30 \mathrm{mph}$ \\
Maximum consecutive outages & 5 & Signal bandwidth & $5 \mathrm{MHz}$ \\
Spreading factor & 16 & Target SINR (at BER=0.1\%) & $8.0 \mathrm{~dB}$ \\
\hline
\end{tabular}

Table 1: Simulation parameters.

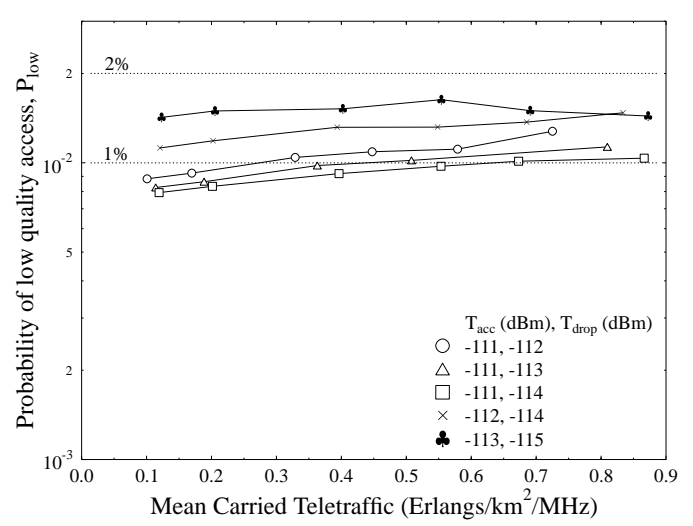

Figure 3: Probability of low quality access versus mean carried traffic of a CDMA based cellular network using fixed received pilot power based soft handover thresholds in conjunction with $0.5 \mathrm{~Hz}$ shadowing having a standard deviation of $3 \mathrm{~dB}$ for $\mathrm{SF}=16$.

nient scenario's set of criteria, which consists of a maximum call dropping probability of $1 \%$ and a probability of low quality access of below $2 \%$, using the thresholds of $T_{a c c}=-$ $113 \mathrm{dBm}$ and $T_{\text {drop }}=-115 \mathrm{dBm}$, respectively. A range of further system performance figures are summarised in Table 2 .

\subsection{Relative Received Pilot Power Based Handovers}

Based on the relatively poor performance of the fixed pilot threshold based scenarios of the previous subsection the importance of employing relative received pilot power thresholds cannot be over-emphasized in realistic propagation environments exposed to shadow fading. More explicitly, in contrast to the previously used fixed thresholds, which were expressed in terms of $\mathrm{dBm}$, i.e. with respect to $1 \mathrm{~mW}$, when using relative thresholds the values of $T_{a c c}$ and $T_{\text {drop }}$ are expressed in terms of $\mathrm{dB}$ relative to the received pilot strength of the base stations in the ABS. The employ- ment of relative thresholds also caters for situations, where the absolute pilot power may be too low for use in conjunction with fixed thresholds, but nonetheless sufficiently high for reliable communications. Similarly to the fixedthreshold based scenario, a range of system performance results are summarised in Table 2 .

\section{3. $E_{c} / I_{o}$ Power Based Soft Handovers}

An alternative soft handover metric used to determine "cell ownership" is the pilot to downlink interference ratio of a cell, which is denoted by $E_{c} / I_{o}$. This handover metric was proposed for employment in the 3rd generation systems [10]. The pilot to downlink interference ratio, or $E_{c} / I_{o}$, may be calculated thus as [11]:

$$
\frac{E_{c}}{I_{o}}=\frac{P_{\text {pilot }}}{P_{\text {pilot }}+N_{0}+\sum_{k=1}^{N_{c e l l s}} P_{k} T_{k}},
$$

where $P_{k}$ is the total transmit power of cell $k, T_{k}$ is the transmission gain which includes antenna gain and pathloss as well as shadowing, $N_{0}$ is the thermal noise and $N_{\text {cells }}$ is the number of cells in the network. The advantage of using such a scheme is that it is not an absolute measurement that is used, but the ratio of the pilot power to the interference power. Thus, if fixed thresholds were used a form of admission control may be employed for new calls if the interference level became too high. A further advantage of this technique is that it takes into account the time-varying nature of the interference level in a shadowed environment. The various system performance figures are summarised in Table 2.

\subsection{Relative $E_{c} / I_{o}$ Threshold Based Handovers}

Finally, we also attempted to combine the benefits of using the received $E_{c} / I_{o}$ ratio and relative soft handover thresholds, thus ensuring that variations in both the received pilot signal strength and interference levels were monitored in the soft handover process. In the next subsection we will compare the performance of a network employing the above four different handover regimes. 


\begin{tabular}{|c|c|c|c|c|c|c|c|c|c|}
\hline \multirow{2}{*}{$\begin{array}{l}\text { Soft handover } \\
\text { algorithm }\end{array}$} & \multirow[b]{2}{*}{ Shadowing } & \multicolumn{4}{|c|}{$\begin{array}{l}\text { Conservative scenario } \\
P_{F T}=1 \%, P_{\text {low }}=1 \% \\
\qquad \text { Power }(\mathrm{dBm})\end{array}$} & \multicolumn{4}{|c|}{$\begin{array}{r}\text { Lenient scenario } \\
P_{F T}=1 \%, P_{l o w}=2 \% \\
\mid \text { Power }(\mathrm{dBm})\end{array}$} \\
\hline & & Users & $\mathrm{ABS}$ & MS & $\mathrm{BS}$ & Users & $\mathrm{ABS}$ & MS & $\mathrm{BS}$ \\
\hline Fixed pilot pwr. & No & 290 & 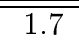 & 5.1 & $\overline{5.1}$ & 290 & 1.7 & 5.1 & 5.1 \\
\hline Fixed pilot pwr. & $0.5 \mathrm{~Hz}, 3 \mathrm{~dB}$ & - & - & - & - & 127 & 1.83 & -2.0 & 6.5 \\
\hline Fixed pilot pwr. & $1.0 \mathrm{~Hz}, 3 \mathrm{~dB}$ & - & - & - & - & - & - & - & - \\
\hline Relative pilot pwr. & No & 288 & 1.7 & 4.1 & 4.7 & 288 & 1.7 & 4.1 & 4.1 \\
\hline Relative pilot pwr. & $0.5 \mathrm{~Hz}, 3 \mathrm{~dB}$ & 144 & 1.77 & -1.5 & 0.6 & 146 & 1.78 & -1.5 & 1.3 \\
\hline Relative pilot pwr. & $1.0 \mathrm{~Hz}, 3 \mathrm{~dB}$ & 127 & 1.5 & -2.4 & -1.9 & 144 & 1.72 & -1.5 & 0.8 \\
\hline Fixed $E_{c} / I_{o}$ & No & 223 & 1.83 & 2.0 & 10.0 & 231 & 1.86 & 2.0 & 10.3 \\
\hline Fixed $E_{c} / I_{o}$ & $0.5 \mathrm{~Hz}, 3 \mathrm{~dB}$ & 129 & 1.88 & -2.4 & 7.0 & 140 & 1.91 & -2.4 & 8.7 \\
\hline Fixed $E_{c} / I_{o}$ & $1.0 \mathrm{~Hz}, 3 \mathrm{~dB}$ & 107 & 1.86 & -3.0 & 4.5 & 128 & 1.91 & -3.0 & 9.5 \\
\hline Relative $E_{c} / I_{o}$ & No & 256 & 1.68 & 3.1 & 2.7 & 256 & 1.68 & 3.1 & 2.7 \\
\hline Relative $E_{c} / I_{o}$ & $0.5 \mathrm{~Hz}, 3 \mathrm{~dB}$ & $\approx 150$ & 1.65 & -1.2 & -1.7 & $\approx 150$ & 1.65 & -1.2 & -1.7 \\
\hline Relative $E_{c} / I_{o}$ & $1.0 \mathrm{~Hz}, 3 \mathrm{~dB}$ & 144 & 1.65 & -1.1 & -1.6 & 144 & 1.65 & -1.1 & -1.6 \\
\hline
\end{tabular}

Table 2: Maximum number of mobile users that can be supported by the network, for different soft handover metrics/algorithms whilst meeting the preset quality constraints. The mean number of base stations in the Active Base station Set (ABS) is also presented, along with the mean mobile and mean base station transmit powers.

\subsection{Performance Overview and Conclusions}

Due to lack of space we cannot present the corresponding results in diagrammatic form for all the previously mentioned performance metrics and for all investigated system scenarios. Instead, Table 2 summarises the results obtained for the various soft handover algorithms over the three different propagation environments considered. The fixed receiver pilot power based algorithm performed the least impressively overall, as expected due to its inherent inability to cope with shadow fading. However, it did offer a high network capacity in a non-shadowed environment. Using the relative received pilot power based soft handover algorithm improved the performance under shadow fading, but different fading rates required different thresholds to meet the conservative and lenient quality criteria. The performance of the fixed $E_{c} / I_{o}$ based soft handover algorithm also varied significantly, when using the same thresholds for the two different fading rates considered. However, the maximum network capacity achieved under the different shadow fading conditions was significantly higher, than that of the fixed received pilot power based algorithm. This benefit resulted from the inclusion of the interference levels in the handover process, which thus took into account the fading of both the signal and the co-channel interference. Combining the relative threshold based scheme with using $E_{c} / I_{o}$ thresholds allowed us to support the highest number of users under the shadow fading conditions investigated. Whilst its performance was not the highest in the non-shadowed environment, this non-shadowed propagation environment is often unrealistic. Hence, due to its best performance in shadowed environments, the relative received $E_{c} / I_{o}$ based soft handover algorithm was our best candidate in conjunction with the soft handover thresholds of $T_{a c c}=-10 \mathrm{~dB}$ and $T_{\text {drop }}=-18 \mathrm{~dB}$. The advantages of this handover algorithm were its reduced fraction of time spent in soft handover, and its ability to perform well under both shadow fading conditions, whilst utilising the same soft handover thresholds.

\section{REFERENCES}

[1] R. Prasad, CDMA for Wireless Personal Communications. Artech House, Inc., 1996.

[2] R. Steele and L. Hanzo, Mobile Radio Communications. IEEE Press - John Wiley, 2nd ed., 1999.

[3] A. J. Viterbi, CDMA: Principles of Spread Spectrum Communication. Addison-Wesley, June 1995. ISBN 0201633744.

[4] T. Ojanperä and R. Prasad, Wideband CDMA for Third Generation Mobile Communications. Artech House, Inc., 1998.

[5] M. K. Simon, J. K. Omura, R. A. Scholtz, and B. K. Levitt, Spread Spectrum Communications Handbook. McGraw Hill, 1994.

[6] S. Verdú, Multiuser Detection. Cambridge University Press, 1998.

[7] M. M. L. Cheng and J. C. I. Chuang, "Performance evaluation of distributed measurement-based dynamic channel assignment in local wireless communications," IEEE JSAC, vol. 14, pp. 698-710, May 1996.

[8] P. J. Cherriman, E. L. Kuan, and L. Hanzo, "Burst-byburst adaptive joint-detection CDMA/H.263 based video telephony," in Proceedings of the ACTS Mobile Communications Summit, Sorrento, Italy, pp. 715-720, June 1999.

[9] J.S. Blogh, P.J. Cherriman, L. Hanzo: Dynamic Channel Allocation Techniques Using Adaptive Modulation and Adaptive Antennas, IEEE JSAC, January 2001

[10] H. Holma and A. Toskala, eds., WCDMA for UMTS : Radio Access for Third Generation Mobile Communications. John Wiley \& Sons, Ltd., 2000.

[11] R. Owen, P. Jones, S. Dehgan, and D. Lister, "Uplink WCDMA capacity and range as a function of inter-to-intra cell interference: theory and practice," in IEEE Proceedings of Vehicular Technology Conference, vol. 1, (Tokyo, Japan), pp. 298-303, 2000. 